\title{
Existence of least energy sign-changing solution for the nonlinear Schrödinger system with two types of nonlocal terms
}

\section{Yuhua Li and Yawen Hao*}

"Correspondence: yhli@sxu.edu.cn School of Mathematical Sciences, Shanxi University, Taiyuan, Shanxi 030006, P.R. China

\section{Springer}

\begin{abstract}
In the paper, we are concerned with the system of Kirchhoff-Schrödinger-Poisson system under certain assumptions on $V_{1}, V_{2}, K$ and $f$. We are interested in the existence of least energy sign-changing solutions to the system on $\mathbb{R}^{N}$. Because two kinds of nonlocal terms $\phi_{u}$ and $\int_{\mathbb{R}^{N}}|\nabla u|^{2}$ are involved in the system, the methods are different from the Kirchhoff or the Schrödinger-Poisson system. The two nonlocal terms $\int_{\mathbb{R}^{N}}|\nabla u|^{2}$ and $\phi_{u}$ make that the functional $J\left(u^{+}+u^{-}\right) \neq J\left(u^{+}\right)+J\left(u^{-}\right)$. Moreover, the nonlocal term $\phi_{u}$ does not have the convergence property because of the assumption $V_{2}$. In addition, the convergence of these two nonlocal terms are different. In the present paper, we unify the increasing property conditions on sign-changing solution in previous papers. We construct a new homotopy operator and then weaken the assumption that $f$ is $C^{1}$ to that of $f$ being only continuous. We prove that the system has a sign-changing solution via a constraint variational method combining with Brouwer's degree theory.
\end{abstract}

MSC: 47J30; 34B15

Keywords: Schrödinger-Poisson system; Kirchhoff; sign-changing solution; Brouwer's degree; constraint variational

\section{Introduction}

In this paper, we consider the nonlinear Kirchhoff-Schrödinger-Poisson system

$$
\begin{cases}-\left(1+b \int_{\mathbb{R}^{N}}|\nabla u|^{2}\right) \Delta u+V_{1}(x) u+V_{2}(x) \phi u=K(x) f(u), & x \in \mathbb{R}^{N}, \\ -\Delta \phi=V_{2}(x) u^{2}, & x \in \mathbb{R}^{N},\end{cases}
$$

for $3 \leq N \leq 5$. System (1.1) with $b=0$ has been introduced while looking for the existence of standing waves for the Schrödinger equation acting with an electrostatic field. We refer to [1] and the references therein for more details as regards the physics aspects. This problem is called the Schrödinger-Poisson system. If $V_{2}=0$, system (1.1) is the Kirchhoff equation, which is the stationary problem associated to the time-dependent problem, which models small vertical vibrations of an elastic string [2].

In recent years, Schrödinger equations with nonlocal terms have attracted much attention. There are several nonlocal Schrödinger equations such as the Kirchhoff and the Schrödinger-Poisson system, and fractional order differential equations. Because of the

(c) The Author(s) 2016. This article is distributed under the terms of the Creative Commons Attribution 4.0 International License (http://creativecommons.org/licenses/by/4.0/), which permits unrestricted use, distribution, and reproduction in any medium, provided you give appropriate credit to the original author(s) and the source, provide a link to the Creative Commons license, and indicate if changes were made. 
nonlocal effect, the convergence and energy functional in a variational reduction are different from local ones. There have been papers studying fractional order differential equations; see [3-9]. When $b=0,(1.1)$ is the Schrödinger-Poisson system. While $V_{2}=0$, (1.1) is the famous Kirchhoff equation. There has been interest in studying problem (1.1) with $b=0$ or $V_{2}=0$ under various assumptions on $K$ and $V_{1}$ or $V_{2}$. For $b=0, K(x)=1$, the existence of positive solutions, ground state solutions, and multiple solutions for (1.1) has been massively addressed in the mathematical literature [10-14]. When $V_{2}=0$, there have been many papers to study the existence of positive solution and infinitely many solutions; see [15-20]. Especially, [16] studied the existence of nonnegative solutions to critical Kirchhoff problem. In addition, the systems with two types of nonlocal terms has been studied; see $[8,21]$. In [8], fractional $p$-Kirchhoff equations was considered. The sign-changing solutions to Kirchhoff problems are also considered. For example, in [22-24], the authors proved the existence of sign-changing solutions for $b=0, V_{2}(x)=K(x)=1$. When $V_{2}=0$, [25] studied the existence of the least energy sign-changing solution to (1.1) with $V_{1}(x)=K(x)=1$. However, there have appeared few papers to study the case of $b \neq 0$ and $V_{2} \neq 0$; see [21]. In [21], the authors considered infinitely many solutions to (1.1).

Motivated by the above work, the main aim of this paper is to study the existence of a sign-change of (1.1) when the potentials $V_{1}$ and $K$ decay to zero as $|x| \rightarrow \infty$. Precisely, we suppose:

(V) $V_{1}: \mathbb{R}^{N} \rightarrow \mathbb{R}$ is a smooth function and there exist $a, c>0$ and $\tau \in(0,2)$ such that

$$
\frac{a}{1+|x|^{\tau}} \leq V_{1}(x) \leq c, \quad \forall x \in \mathbb{R}^{N}
$$

and $V_{2} \in L^{\infty}\left(\mathbb{R}^{N}\right) \cup L^{(6-N) / 2 N}\left(\mathbb{R}^{N}\right)$ is nonnegative;

(K) $K: \mathbb{R}^{N} \rightarrow \mathbb{R}$ is a smooth function and there exist $\xi>\tau, d>0$ such that

$$
0<K(x) \leq \frac{d}{1+|x|^{\xi}}, \quad \forall x \in \mathbb{R}^{N}
$$

As regards the function $f$, we assume $f \in C(\mathbb{R}, \mathbb{R})$ and we have the following hypotheses:

$\left(\mathrm{f}_{1}\right) f(t)=o(|t|)$ as $t \rightarrow 0$;

$\left(\mathrm{f}_{2}\right) \lim _{t \rightarrow \infty} \frac{f(t)}{t^{3}}=+\infty$, where $F(t)=\int_{0}^{u} f(t) d t$;

$\left(\mathrm{f}_{3}\right)$ there exists a $\theta \in[0,1)$ such that $\frac{K(x) f(t)-\theta V(x) t}{|t|^{3}}$ is nondecreasing on $(-\infty, 0)$ and $(0, \infty)$, respectively;

$\left(\mathrm{f}_{4}\right) \quad f(t) \mid \leq C\left(|t|+|t|^{p}\right), p>3$.

The conditions $\left(\mathrm{f}_{1}\right)-\left(\mathrm{f}_{4}\right)$ with $\theta=0$ are usual for the Kirchhoff equation or the Schrödinger-Poisson system; see $[22,25]$. The condition $\left(f_{3}\right)$ is equivalent to the one in $[26$, 27]

$\left(\mathrm{V}^{\prime}\right) K(x)\left[\frac{f(\tau)}{\tau^{3}}-\frac{f(t \tau)}{(t \tau)^{3}}\right] \operatorname{sign}(1-t)+\theta V(x) \frac{\left|1-t^{2}\right|}{(t \tau)^{2}} \geq 0, t>0, \tau \neq 0$.

In fact, we have the next remark.

Remark 1.1 Condition $\left(f_{3}\right)$ is equivalent to $\left(V^{\prime}\right)$. 
Proof We only prove the case of $t>0$. For $t \in(0,1], t \tau \leq \tau$, then

$$
\begin{gathered}
K(x)\left[\frac{f(\tau)}{\tau^{3}}-\frac{f(t \tau)}{(t \tau)^{3}}\right] \operatorname{sign}(1-t)+\theta V(x) \frac{\left|1-t^{2}\right|}{(t \tau)^{2}} \\
=K(x)\left[\frac{f(\tau)}{\tau^{3}}-\frac{f(t \tau)}{(t \tau)^{3}}\right]+\theta V(x) \frac{1-t^{2}}{(t \tau)^{2}} \\
=\frac{K(x) f(\tau)-\theta V(x) \tau}{\tau^{3}}-\frac{K(x) f(t \tau)-\theta V(x) t \tau}{(t \tau)^{3}} .
\end{gathered}
$$

If $t>1$, then by $t \tau \geq \tau$, we have

$$
\begin{gathered}
K(x)\left[\frac{f(\tau)}{\tau^{3}}+\frac{f(t \tau)}{(t \tau)^{3}}\right] \operatorname{sign}(1-t)+\theta V(x) \frac{\left|1-t^{2}\right|}{(t \tau)^{2}} \\
=-K(x)\left[\frac{f(\tau)}{\tau^{3}}+\frac{f(t \tau)}{(t \tau)^{3}}\right]-\theta V(x) \frac{1-t^{2}}{(t \tau)^{2}} \\
=-\frac{K(x) f(\tau)-\theta V(x) \tau}{\tau^{3}}+\frac{K(x) f(t \tau)-\theta V(x) t \tau}{(t \tau)^{3}} .
\end{gathered}
$$

Therefore, the conclusion holds.

In addition, we must notice that the condition on $V_{2}$ cannot ensure the nonlocal term $\phi_{u_{n}} \rightarrow \phi_{u}$ if $u_{n} \rightarrow u$ in $L^{2 N /(N-2)}$. The least energy sign-changing solution to the KirchhoffSchrödinger-Poisson system has not been studied under the conditions (V) and (K). We unify the conditions $\theta=0$ and $\theta>0$ and generalize to the problem (1.1). Throughout this paper, we consider the weighted space $L_{K}^{q}$ of measurable $u: \mathbb{R}^{N} \rightarrow \mathbb{R}$ such that

$$
|u|_{q, K}:=\left[\int_{\mathbb{R}}^{N} K(x)|u(x)|^{q} d x\right]^{\frac{1}{q}}<\infty .
$$

If $K$ is a constant, $L_{K}^{p}$ is the usual space $L^{p}$ and the norm is denoted by $|\cdot|_{p}$. The weighted Sobolev spaces $E$ defined by setting

$$
E=\left\{u \in D^{1,2}\left(\mathbb{R}^{N}\right): \int_{\mathbb{R}}^{N} V_{1}(x) u^{2} d x<+\infty\right\}
$$

make $E$ a Hilbert space with the inner product and the norm

$$
(u, v)=\int_{\mathbb{R}}^{N}\left(\nabla u \cdot \nabla v+V_{1}(x) u v\right) d x, \quad\|u\|=(u, u)^{\frac{1}{2}}
$$

Then we have the following embedding theorem, which is often used later.

Proposition 1.2 ([28]) Let $N \geq 3$ and suppose that (V), (K) hold with $\tau \in(0,2]$ and $\xi>0$, respectively. Then, for $p \in\left[1, \frac{N+2}{N-2}\right]$, there is $C>0$ such that

$$
|u|_{p+1, K} \leq C\|u\|, \quad u \in E .
$$

Furthermore, the embedding $E \hookrightarrow L_{K}^{p+1}$ is compact for $p \in\left(1, \frac{N+2}{N-2}\right)$. 
Let $g(x, t)=K(x) f(t)-\theta V(x) t$ and $G(x, t)=\int_{0}^{t} g(x, s) d s$, then we must notice the following fact.

Remark 1.3 If $u_{n} \rightarrow u$ in $E$, then $\int_{\mathbb{R}^{3}} K F\left(u_{n}\right) \rightarrow \int_{\mathbb{R}^{3}} K F(u)$ and $\int_{\mathbb{R}^{3}} K f\left(u_{n}\right) u_{n} \rightarrow \int_{\mathbb{R}^{3}} K f(u) u$, for the proof see Lemma 2.1. However, $G(x, t)$ and $g(x, t)$ do not satisfy these properties, due to the term $\int_{\mathbb{R}^{3}} V(x) u_{n}^{2}$. Therefore, we cannot replace $K f(u)-\theta V(x) u$ with $g(x, u)$.

For every $u \in E$, the Lax-Milgram theorem implies that there exists a unique $\phi_{u} \in$ $D^{1,2}\left(\mathbb{R}^{N}\right)$, satisfying $-\Delta \phi_{u}=V_{2} u^{2}$. Thus, we can define the corresponding functional to (1.1) as follows:

$$
\begin{aligned}
J(u) & :=\frac{1}{2} \int_{\mathbb{R}^{N}}\left(|\nabla u|^{2}+V_{1}(x) u^{2}\right)+\frac{b}{4}\left(\int_{\mathbb{R}^{N}}|\nabla u|^{2}\right)^{2}+\frac{1}{4} \int_{\mathbb{R}^{N}} V_{2}(x) \phi_{u} u^{2}-\int_{\mathbb{R}^{N}} K F(u) \\
& =\frac{1}{2}\|u\|^{2}+\frac{b}{4}\left(\int_{\mathbb{R}^{N}}|\nabla u|^{2}\right)^{2}+\frac{1}{4} \int_{\mathbb{R}^{N}} V_{2}(x) \phi_{u} u^{2}-\int_{\mathbb{R}^{N}} K F(u) .
\end{aligned}
$$

Here $G(x, u)=\int_{0}^{u} g(x, t) d t$. It is obvious that $J \in C^{1}(E, \mathbb{R})$ and

$$
\begin{aligned}
\left\langle J^{\prime}(u), \varphi\right\rangle= & \int_{\mathbb{R}^{N}}\left(\nabla u \nabla \varphi+V_{1}(x) u \varphi\right)+b \int_{\mathbb{R}^{N}}|\nabla u|^{2} \int_{\mathbb{R}^{N}} \nabla u \cdot \nabla \varphi \\
& +\int_{\mathbb{R}^{N}} V_{2}(x) \phi_{u} u \varphi-\int_{\mathbb{R}^{N}} K f(u) \varphi \\
= & (u, \varphi)+b \int_{\mathbb{R}^{N}}|\nabla u|^{2} \int_{\mathbb{R}^{N}} \nabla u \cdot \nabla \varphi+\int_{\mathbb{R}^{N}} V_{2}(x) \phi_{u} u \varphi-\int_{\mathbb{R}^{N}} K(x) f(u) \varphi .
\end{aligned}
$$

Clearly, critical points of $J$ are the weak solutions to nonlocal problem (1.1). Furthermore, if $u \in E$ is a solution of (1.1) and $u^{ \pm} \neq 0$, then $u$ is sign-changing, where

$$
u^{+}(x)=\max \{u(x), 0\} \quad \text { and } \quad u^{-}(x)=\min \{u(x), 0\}
$$

In order to get a least energy sign-changing solution for the system (1.1), we first try to seek a minimizer of the energy functional $J$ with the following constraint:

$$
M:=\left\{u \in E: u^{ \pm} \neq 0,\left\langle J^{\prime}(u), u^{+}\right\rangle=\left\langle J^{\prime}(u), u^{-}\right\rangle=0\right\}
$$

and then we show that the minimizer is a sign-changing solution of (1.1). But in our problem (1.1), since $f$ is not $C^{1}$, which is assumed in [22, 25], it is rather difficult to show that $M \neq \emptyset$. Thus, we must introduce some new ideas to get a sign-changing solution for the problem (1.1). In the present paper, we use a new homotopy operator which is $C^{1}$.

Now, we state our main theorem.

Theorem 1.4 If we have the assumptions $(\mathrm{V}),(\mathrm{K})$, and $\left(\mathrm{f}_{1}\right)-\left(\mathrm{f}_{4}\right)$, then the problem (1.1) possesses at least one sign-changing solution which is the least energy sign-changing solution.

The paper is organized as follows. In Section 2, we prove several lemmas, which are crucial to prove our main results. In Section 3, we show that the minimizer of the constrained problem is a sign-changing solution by a new homotopy operator. 


\section{Preliminaries}

Firstly, we give the convergence of the nonlinear term.

Lemma 2.1 Assume that $u_{n} \rightarrow u$ in $E$, then $\int_{\mathbb{R}^{N}} K(x) f\left(u_{n}\right) u_{n} \rightarrow \int_{\mathbb{R}^{N}} K(x) f(u) u$ and $\int_{\mathbb{R}^{3}} K(x) F\left(u_{n}\right) \rightarrow \int_{\mathbb{R}^{3}} K(x) F(u)$.

Proof By Proposition 1.2, $u_{n} \rightarrow u$ strongly in $L_{K}^{q}$ for $q \in[2,6)$. Thus after passing to a subsequence, $u_{n}(x) \rightarrow u(x)$ for a.e. $x \in \mathbb{R}^{N}$. From the convergence of $u_{n} \rightarrow u$ in $L_{K}^{2}$ and $L_{K}^{p+1}$, there exist two functions $a \in L^{2}$ and $b \in L^{p+1}$ such that $K(x) u_{n}^{2} \leq a^{2}(x)$ and $K(x)\left|u_{n}\right|^{p+1} \leq$ $[b(x)]^{p+1}$ for a.e. $x \in \mathbb{R}^{N}$. Therefore, $\left(\mathrm{f}_{4}\right)$ implies that

$$
\begin{aligned}
\left|\int_{\mathbb{R}^{N}} K(x) f\left(u_{n}\right) u_{n}\right| & \leq\left.\int_{\mathbb{R}^{N}} C|K(x)| u_{n}\right|^{2}+C K(x)\left|u_{n}\right|^{p+1} \\
& \leq \int_{\mathbb{R}^{N}}\left[C(a(x))^{2}+C(b(x))^{p+1}\right] d x .
\end{aligned}
$$

According to the dominated convergence theorem, $\int_{\mathbb{R}^{3}} K(x) f\left(u_{n}\right) u_{n} \rightarrow \int_{\mathbb{R}^{3}} K(x) f(u) u$. A similar argument implies that $\int_{\mathbb{R}^{3}} K(x) F\left(u_{n}\right) \rightarrow \int_{\mathbb{R}^{3}} K(x) F(u)$. The proof is completed.

The next lemma shows that $M \neq \emptyset$.

Lemma 2.2 Assume that $\left(\mathrm{f}_{1}\right)-\left(\mathrm{f}_{4}\right)$ hold. If $u \in E$ with $u^{ \pm} \neq 0$, then there is a unique pair $(t, s)$ of positive numbers such that $t u^{+}+s u^{-} \in M$.

Proof Fix $u \in E$ with $u^{ \pm} \neq 0, \int_{\mathbb{R}^{N}} \nabla \phi_{u^{+}} \cdot \nabla \phi_{u^{-}}=\int_{\mathbb{R}^{N}} V_{2}(x) \phi_{u^{+}}\left(u^{-}\right)^{2}$; since $-\Delta \phi_{u^{-}}=$ $V_{2}(x)\left(u^{-}\right)^{2}$, we can obtain

$$
\int_{\mathbb{R}^{N}} V_{2}(x) \phi_{u^{+}}\left|u^{-}\right|^{2} d x=\int_{\mathbb{R}^{N}} V_{2}(x) \phi_{u^{-}}\left|u^{+}\right|^{2} d x=\int_{\mathbb{R}^{N}} \nabla \phi_{u^{+}} \cdot \nabla \phi_{u^{-}} .
$$

Denote $A:=b \int_{\mathbb{R}^{N}}\left|\nabla u^{+}\right|^{2} d x \int_{\mathbb{R}^{N}}\left|\nabla u^{-}\right|^{2} d x+\int_{\mathbb{R}^{N}} V_{2}(x) \phi_{u^{-}}\left|u^{+}\right|^{2} d x$ for convenience. We introduce the following notations:

$$
\begin{array}{ll}
a_{1}=\left\|u^{+}\right\|^{2}, & a_{2}=b\left(\int_{\mathbb{R}^{N}}\left|\nabla u^{+}\right|^{2}\right)^{2} ; \\
b_{1}=\left\|u^{-}\right\|^{2}, & b_{2}=b\left(\int_{\mathbb{R}^{N}}\left|\nabla u^{-}\right|^{2}\right)^{2} .
\end{array}
$$

Since $-\Delta \phi_{u}=V_{2}(x) u^{2}=V_{2}(x)\left(u^{+}\right)^{2}+V_{2}(x)\left(u^{-}\right)^{2}=-\Delta\left(\phi_{u^{+}}+\phi_{u^{-}}\right), \phi_{u}=\phi_{u^{+}}+\phi_{u^{-}}$. Then, $t u^{+}+s u^{-}$is contained in $M$ if and only if

$$
\left\{\begin{array}{l}
g_{1}(t, s):=a_{1} t^{2}+a_{2} t^{4}+A t^{2} s^{2}-\int_{\mathbb{R}^{N}} K f\left(t u^{+}\right)\left(t u^{+}\right)=0 \\
g_{2}(t, s):=b_{1} s^{2}+b_{2} s^{4}+A t^{2} s^{2}-\int_{\mathbb{R}^{N}} K f\left(s u^{-}\right)\left(s u^{-}\right)=0
\end{array}\right.
$$

We have from $\left(\mathrm{f}_{1}\right),\left(\mathrm{f}_{2}\right)$, and $(2.3) g_{1}(t, t)>0$ and $g_{2}(t, t)>0$ for $t>0$ small and $g_{1}(s, s)<0$, $g_{2}(s, s)<0$ for $s>0$ large. Thus there exist $0<\delta<R$ such that

$$
g_{1}(\delta, \delta)>0, \quad g_{1}(R, R)<0 ; \quad g_{2}(\delta, \delta)<0, \quad g_{2}(R, R)<0 .
$$


From (2.3), it follows that $g_{1}(\delta, s), g_{1}(R, s)$ both are increasing for $s \geq 0$ and $g_{2}(t, R), g_{2}(t, \delta)$ both are increasing for $t \geq 0$. Therefore, by (2.4), we have

$$
g_{1}(\delta, s)>0, \quad g_{1}(R, s)<0, \quad s \in[\delta, R]
$$

and

$$
g_{2}(t, \delta)>0, \quad g_{2}(t, R)<0, \quad t \in[\delta, R] .
$$

By (2.5), (2.6), and Miranda's theorem [29], there exists a point $\left(t_{u}, s_{u}\right)$ with $t_{u}, s_{u} \in(\delta, R)$ such that $g_{1}\left(t_{u}, s_{u}\right)=g_{2}\left(t_{u}, s_{u}\right)=0$.

In the following, we prove the uniqueness of $(t, s)$. If there are two pairs $\left(t_{1}, s_{1}\right)$ and $\left(t_{2}, s_{2}\right)$ such that $t_{1} u^{+}+s u^{-} \in M$ and $t_{2} u^{+}+s u^{-} \in M$, then $\left(t_{1} / t_{2}\right) t_{2} u^{+}+\left(s_{1} / s_{2}\right) s_{2} u^{-} \in M$. Therefore, we may assume that $u \in M$ and $t u^{+}+s u^{-} \in M$. We need only to prove that $t=s=1$. We assume that $0<t \leq s$. Then, by $(2.3)$ and $\theta<1$, we have $b_{1}-\theta \int_{\mathbb{R}^{N}} V(x)\left(u^{-}\right)^{2}>0$ and

$$
\left[b_{1}-\theta \int_{\mathbb{R}^{N}} V(x)\left(u^{-}\right)^{2}\right] \frac{1}{s^{2}}+b_{2}+A \geq \int_{\mathbb{R}^{N}} \frac{\left[K f\left(s u^{-}\right)-\theta V(x) s u^{-}\right]}{\left(s u^{-}\right)^{3}}\left(u^{-}\right)^{4} .
$$

Since $u \in M$,

$$
\begin{aligned}
& {\left[b_{1}-\theta \int_{\mathbb{R}^{N}} V(x)\left(u^{-}\right)^{2}\right]\left[\frac{1}{s^{2}}-1\right]} \\
& \quad \geq \int_{\mathbb{R}^{N}}\left\{\frac{\left[K f\left(s u^{-}\right)-\theta V(x) s u^{-}\right]}{\left(s u^{-}\right)^{3}}-\frac{\left[K f\left(u^{-}\right)-\theta V(x) u^{-}\right]}{\left(u^{-}\right)^{3}}\right\}\left(u^{-}\right)^{4} .
\end{aligned}
$$

According to condition $\left(\mathrm{f}_{3}\right), s \leq 1$. On the other hand, since $t \leq s$ and $u \in M$, similarly, we can obtain

$$
\begin{aligned}
& {\left[a_{1}-\theta \int_{\mathbb{R}^{N}} V(x)\left(u^{+}\right)^{2}\right]\left[\frac{1}{t^{2}}-1\right]} \\
& \quad \leq \int_{\mathbb{R}^{N}}\left\{\frac{\left[K f\left(t u^{+}\right)-\theta V(x) t u^{+}\right]}{\left(t u^{+}\right)^{3}}-\frac{\left[K f\left(u^{+}\right)-\theta V(x) u^{+}\right]}{\left(u^{+}\right)^{3}}\right\}\left(u^{+}\right)^{4} .
\end{aligned}
$$

Therefore, $t \geq 1$. Consequently, $t=s=1$.

Lemma 2.3 For $u \in E$ with $u^{ \pm} \neq 0$, the pair $\left(t_{u}, s_{u}\right)$ obtained in Lemma 2.2 is the maximum point of the function $\psi: \mathbb{R}_{+} \times \mathbb{R}_{+} \rightarrow \mathbb{R}$ defined as $\psi(t, s)=J\left(t u^{+}+s u^{-}\right)$, where $\mathbb{R}_{+}=[0, \infty)$.

Proof From the proof of Lemma 2.2, $\left(t_{u}, s_{u}\right)$ is a critical point of $\psi$ in $\mathbb{R}_{+} \times \mathbb{R}_{+}$. By assumptions $\left(\mathrm{f}_{1}\right),\left(\mathrm{f}_{2}\right)$, and the proof of Lemma 2.2, we deduce that $J\left(t u^{+}+s u^{-}\right)>0$ for $t, s>0$ small and $\psi(t, s) \rightarrow-\infty$ as $|(t, s)| \rightarrow \infty$. Therefore, $\left(t_{u}, s_{u}\right)$ is the maximum point. In the following, we prove that $t_{u}, s_{u}>0$. It is sufficient to check that a maximum point cannot be achieved on the boundary of $\mathbb{R}_{+} \times \mathbb{R}_{+}$. Without loss of generality, we may assume that $\left(0, s_{0}\right)$ is a maximum point of $\psi$. Then, for $t>0$ small, by condition $\left(f_{1}\right)$,

$$
\begin{aligned}
\psi\left(t, s_{0}\right) & =J\left(t u^{+}+s_{0} u^{-}\right) \\
& =\frac{t^{2}}{2}\left\|u^{+}\right\|^{2}+\frac{t^{4}}{4} b\left|\nabla u^{+}\right|_{2}^{4}+\frac{t^{4}}{4} \int_{\mathbb{R}^{N}} V_{2}(x) \phi_{u^{+}}\left(u^{+}\right)^{2}-\int_{\mathbb{R}^{N}} K(x) F\left(t u^{+}\right) d x
\end{aligned}
$$




$$
\begin{aligned}
& +\frac{t^{2} s_{0}^{2}}{2} b\left|\nabla u^{+}\right|_{2}^{2}\left|\nabla u^{-}\right|_{2}^{2}+\frac{t^{2} s_{0}^{2}}{2} \int_{\mathbb{R}^{N}} V_{2}(x) \phi_{u^{+}}\left(u^{-}\right)^{2} d x \\
& +\frac{s_{0}^{2}}{2}\left\|u^{-}\right\|^{2}+\frac{s_{0}^{4}}{4} b\left|\nabla u^{-}\right|_{2}^{4}+\frac{s_{0}^{4}}{4} \int_{\mathbb{R}^{N}} V_{2}(x) \phi_{u^{-}}\left(u^{-}\right)^{2}-\int_{\mathbb{R}^{N}} K(x) F\left(s_{0} u^{-}\right) d x \\
> & \frac{s_{0}^{2}}{2}\left\|u^{-}\right\|^{2}+\frac{s_{0}^{4}}{4} b\left|\nabla u^{-}\right|_{2}^{4}+\frac{s_{0}^{4}}{4} \int_{\mathbb{R}^{N}} V_{2}(x) \phi_{u^{-}}\left(u^{-}\right)^{2}-\int_{\mathbb{R}^{N}} K(x) F\left(s_{0} u^{-}\right) d x \\
= & \psi\left(0, s_{0}\right) .
\end{aligned}
$$

The pair $\left(0, s_{0}\right)$ is not a maximum point of $\psi$ in $\mathbb{R}_{+} \times \mathbb{R}_{+}$.

\section{Lemma 2.4}

(i) If $\left\langle J^{\prime}(u), u^{ \pm}\right\rangle<0$, then $\left(t_{u}, s_{u}\right) \in(0,1)^{2}$.

(ii) If $u^{ \pm} \neq 0$ is such that $\left\langle J^{\prime}(u), u^{ \pm}\right\rangle \leq 0$, then $\left(t_{u}, s_{u}\right) \in(0,1]^{2}$.

(iii) If $\left\langle J^{\prime}(u), u^{ \pm}\right\rangle>0$, then $\left(t_{u}, s_{u}\right) \in(1, \infty)^{2}$.

Proof Under the assumptions of (i), (ii), and (iii), we have $u^{ \pm} \neq 0$. Let $c_{1}=a_{1}-\theta \times$ $\int_{\mathbb{R}^{N}} V(x)\left(u^{+}\right)^{2}$ and $d_{1}=b_{1}-\theta \int_{\mathbb{R}^{N}} V(x)\left(u^{-}\right)^{2}$, then $c_{1}, d_{1}>0$. Since $t_{u} u^{+}+s_{u} u^{-} \in M$, we have from (2.3)

$$
\begin{aligned}
\left\langle J^{\prime}(u), u^{+}\right\rangle= & c_{1}\left[1-\frac{1}{t_{u}^{2}}\right]+A\left[1-\frac{s_{u}^{2}}{t_{u}^{2}}\right] \\
& -\int_{\mathbb{R}^{N}}\left[\frac{K(x) f\left(u^{+}\right)-\theta V(x)\left(u^{+}\right)}{\left(u^{+}\right)^{3}}-\frac{K(x) f\left(t_{u} u^{+}\right)-\theta V(x)\left(t_{u} u^{+}\right)}{\left(t_{u} u^{+}\right)^{3}}\right]\left(u^{+}\right)^{4}
\end{aligned}
$$

and

$$
\begin{aligned}
\left\langle J^{\prime}(u), u^{-}\right\rangle= & d_{1}\left[1-\frac{1}{s_{u}^{2}}\right]+A\left[1-\frac{t_{u}^{2}}{s_{u}^{2}}\right] \\
& -\int_{\mathbb{R}^{N}}\left[\frac{K(x) f\left(u^{-}\right)-\theta V(x)\left(u^{-}\right)}{\left(u^{-}\right)^{3}}-\frac{K(x) f\left(s_{u} u^{-}\right)-\theta V(x)\left(s_{u} u^{-}\right)}{\left(s_{u} u^{-}\right)^{3}}\right]\left(u^{-}\right)^{4} .
\end{aligned}
$$

For convenience, we introduce some notations as follows:

$$
\begin{aligned}
& D_{11}=c_{1}\left[1-\frac{1}{t_{u}^{2}}\right]+A\left[1-\frac{s_{u}^{2}}{t_{u}^{2}}\right], \\
& D_{12}=\int_{\mathbb{R}^{N}}\left[\frac{K(x) f\left(u^{+}\right)-\theta V(x)\left(u^{+}\right)}{\left(u^{+}\right)^{3}}-\frac{K(x) f\left(t_{u} u^{+}\right)-\theta V(x)\left(t_{u} u^{+}\right)}{\left(t_{u} u^{+}\right)^{3}}\right]\left(u^{+}\right)^{4}, \\
& D_{21}=d_{1}\left[1-\frac{1}{s_{u}^{2}}\right]+A\left[1-\frac{t_{u}^{2}}{s_{u}^{2}}\right], \\
& D_{22}=\int_{\mathbb{R}^{N}}\left[\frac{K(x) f\left(u^{-}\right)-\theta V(x)\left(u^{-}\right)}{\left(u^{-}\right)^{3}}-\frac{K(x) f\left(s_{u} u^{-}\right)-\theta V(x)\left(s_{u} u^{-}\right)}{\left(s_{u} u^{-}\right)^{3}}\right]\left(u^{-}\right)^{4} .
\end{aligned}
$$

Without loss of generality, we may assume that $t_{u} \geq s_{u}$.

(i) If $t_{u} \geq 1$, then $D_{11} \geq 0$ and by the condition $\left(\mathrm{f}_{3}\right)$, we have $D_{12} \leq 0$. It follows from (2.7) that $\left\langle J^{\prime}(u), u^{+}\right\rangle \geq 0$. This is a contradiction. Thus, $\left(t_{u}, s_{u}\right) \in(0,1)^{2}$.

(ii) If $t_{u}>1$, then $D_{11}>0$ and by the condition $\left(f_{3}\right)$, we have $D_{12} \leq 0$. Therefore, $\left\langle J^{\prime}(u), u^{+}\right\rangle>0$. This is a contradiction. Thus, $\left(t_{u}, s_{u}\right) \in(0,1]^{2}$. 
(iii) If $s_{u} \leq 1$, similarly $D_{21} \leq 0$ and $D_{22} \geq 0$. It follows from (2.8) that $\left\langle J^{\prime}(u), u^{-}\right\rangle \leq 0$, which is a contradiction. Thus, $\left(t_{u}, s_{u}\right) \in(1, \infty)^{2}$.

By Lemma 2.3, we may define the minimization problem

$$
m=\inf \{J(u): u \in M\}
$$

Lemma 2.5 Suppose that conditions $\left(\mathrm{f}_{1}\right)-\left(\mathrm{f}_{4}\right)$ hold, then $m>0$ can be achieved.

Proof For any $u \in M$, we have $\left\langle J^{\prime}(u), u\right\rangle=0$. Then we get from conditions $\left(\mathrm{f}_{1}\right)$ and $\left(\mathrm{f}_{4}\right)$

$$
\begin{aligned}
\|u\|^{2} & \leq \int_{\mathbb{R}^{N}}\left(|\nabla u|^{2}+V_{1}(x) u^{2}+V_{2}(x) \phi_{u} u^{2}\right) d x+b|\nabla u|_{2}^{4} \\
& =\int_{\mathbb{R}^{N}} K(x) f(u) u \\
& \leq \varepsilon \int_{\mathbb{R}^{N}} K(x) u^{2}+C_{\varepsilon} \int_{\mathbb{R}^{N}} K(x) u^{p+1} .
\end{aligned}
$$

Then by Proposition 1.2, we can get

$$
\|u\|^{2} \leq C_{1} \varepsilon\|u\|^{2}+C_{2}\|u\|^{p+1}
$$

where $C_{1}, C_{2}>0$ are constants. So there exists a constant $\alpha>0$ such that $\|u\| \geq \alpha$. Moreover, by $\left(\mathrm{f}_{3}\right)$, we have

$$
\left[K(x) f(t) t-\theta V(x) t^{2}\right]-4\left[K(x) F(t)-\theta \frac{1}{2} V(x) t^{2}\right] \geq 0, \quad x \in \mathbb{R}^{N}, t \in \mathbb{R}
$$

Hence, we have

$$
\begin{aligned}
J(u) & =J(u)-\frac{1}{4}\left\langle J^{\prime}(u), u\right\rangle \\
& =\frac{1}{4}\|u\|^{2}+\frac{1}{4} \int_{\mathbb{R}^{N}} K(x)(f(u) u-4 F(u)) \\
& \geq \frac{1}{4}\|u\|^{2}-\frac{1}{4} \theta \int_{\mathbb{R}^{N}} V(x) u^{2} \\
& \geq \frac{1}{4}(1-\theta) \alpha^{2} .
\end{aligned}
$$

This implies that $m \geq \frac{1}{4}(1-\theta) \alpha^{2}>0$.

Let $\left\{u_{n}\right\} \subset M$ be such that $J\left(u_{n}\right) \rightarrow m$. Then $\left\{u_{n}\right\}$ is bounded in $E$, so there exists a subsequence of $\left\{u_{n}\right\}$ and $u \in E$, we may still denote the subsequence by $\left\{u_{n}\right\}$, such that $u_{n} \rightarrow u$ and $u_{n}^{ \pm} \rightarrow u^{ \pm}$weakly in $E$ and $u_{n}(x) \rightarrow u(x)$ a.e. $x \in \mathbb{R}^{N}$. Since $\left\{u_{n}\right\} \in M$, we have $\left\langle J^{\prime}\left(u_{n}\right), u_{n}^{+}\right\rangle=0$. That is,

$$
\left\|u_{n}^{+}\right\|^{2}+b\left|\nabla u_{n}\right|_{2}^{2}\left|\nabla u_{n}^{+}\right|_{2}^{2}+\int_{\mathbb{R}^{N}} V_{2}(x) \phi_{u_{n}}\left(u_{n}^{+}\right)^{2}=\int_{\mathbb{R}^{N}} K f\left(u_{n}^{+}\right) u_{n}^{+} .
$$


This implies from conditions $\left(f_{1}\right)$ and $\left(f_{4}\right)$ that there exists a constant $\rho>0$ such that $\left\|u_{n}^{+}\right\| \geq \rho$ for all $n \in \mathbb{N}$. By (2.10) and Lemma 2.2, we get

$$
0<\rho^{2} \leq\left\|u_{n}^{+}\right\|^{2} \leq \int_{\mathbb{R}^{N}} K f\left(u_{n}^{+}\right) u_{n}^{+}=\int_{\mathbb{R}^{N}} K f\left(u^{+}\right) u^{+}+o(1),
$$

where $o(1)$ denotes a quantity tending to zero as $n \rightarrow+\infty$. Thus, $u^{+} \neq 0$. Similarly, we have $u^{-} \neq 0$.

Since $u_{n} \rightarrow u$ in $E$, we have $\phi_{u_{n}} \rightarrow \phi_{u}$ in $D^{1,2}\left(\mathbb{R}^{N}\right)$ if $V_{2} \in L^{\infty}\left(\mathbb{R}^{N}\right)$ or $\phi_{u_{n}} \rightarrow \phi_{u}$ in $D^{1,2}\left(\mathbb{R}^{N}\right)$ if $V_{2} \in L^{(6-N) / 2 N}\left(\mathbb{R}^{N}\right)$. By the weak lower semicontinuity of the norm and Fatou's lemma, we have

$$
\begin{aligned}
& \left\|u^{+}\right\|^{2}+b|\nabla u|_{2}^{2}\left|\nabla u^{+}\right|_{2}^{2}+\int_{\mathbb{R}^{N}} V_{2}(x) \phi_{u}\left|u^{+}\right|^{2} d x \\
& \quad \leq \liminf _{n \rightarrow+\infty}\left(\left\|u_{n}^{+}\right\|^{2}+b\left|\nabla u_{n}\right|_{2}^{2}\left|\nabla u_{n}^{-}\right|_{2}^{2}+\int_{\mathbb{R}^{N}} V_{2}(x) \phi_{u_{n}}\left|u_{n}^{+}\right|^{2} d x\right) .
\end{aligned}
$$

Then from (2.10) and Lemma 2.1 we get

$$
\left\|u^{+}\right\|^{2}+b|\nabla u|_{2}^{2}\left|\nabla u^{+}\right|_{2}^{2}+\int_{\mathbb{R}^{N}} V_{2}(x) \phi_{u}\left|u^{+}\right|^{2} d x \leq \int_{\mathbb{R}^{N}} K f\left(u^{+}\right) u^{+} .
$$

Similarly, we have

$$
\left\|u^{-}\right\|^{2}+b|\nabla u|_{2}^{2}\left|\nabla u^{-}\right|_{2}^{2}+\int_{\mathbb{R}^{N}} V_{2}(x) \phi_{u}\left|u^{-}\right|^{2} d x \leq \int_{\mathbb{R}^{N}} K f\left(u^{-}\right) u^{-} .
$$

From (2.11), (2.12), and the proof of Lemma 2.4, there exists $(\bar{t}, \bar{s}) \in(0,1] \times(0,1]$ such that $\bar{u}=\bar{t} u^{+}+\bar{s} u^{-} \in M$. Moreover, according to condition $\left(\mathrm{f}_{3}\right)$ we have $\left[K(x) f(t) t-\theta V(x) t^{2}\right]-$ $4\left[K(x) F(t)-\frac{1}{2} \theta V(x) t^{2}\right] \geq 0$ is nondecreasing on $(-\infty, 0)$ and $(0, \infty)$, respectively. Thus,

$$
\begin{aligned}
m & \leq J(\bar{u})=J(\bar{u})-\frac{1}{4}\left\langle J^{\prime}(\bar{u}), \bar{u}\right\rangle \\
& =\frac{1}{4}\|\bar{u}\|^{2}+\frac{1}{4} \int_{\mathbb{R}^{N}} K(f(\bar{u}) \bar{u}-4 F(\bar{u})) d x \\
& =\frac{1}{4}\left(\left\|\bar{t} u^{+}\right\|^{2}+\left\|\bar{s} u^{-}\right\|^{2}\right)+\frac{1}{4} \int_{\mathbb{R}^{N}} K(f(\bar{u}) \bar{u}-4 F(\bar{u})) d x \\
& \leq \frac{1}{4}|\nabla u|_{2}^{2}+\frac{1}{4} \int_{\mathbb{R}^{N}} V(x) \bar{u}^{2}+\frac{1}{4} \int_{\mathbb{R}^{N}} K(f(u) u-4 F(u)) d x+\frac{1}{4} \theta \int_{\mathbb{R}^{N}} V(x)\left[u^{2}-\bar{u}^{2}\right] \\
& \leq \frac{1}{4}|\nabla u|_{2}^{2}+\frac{1}{4}(1-\theta) \int_{\mathbb{R}^{N}} V(x) u^{2}+\frac{1}{4} \int_{\mathbb{R}^{N}}\left[K(f(u) u-4 F(u))+\theta V(x) u^{2}\right] \\
& \leq \liminf _{n \rightarrow \infty}\left[J\left(u_{n}\right)-\frac{1}{4}\left\langle J^{\prime}\left(u_{n}\right), u_{n}\right\rangle\right]=m .
\end{aligned}
$$

By the above inequality and Lemma 2.3 we deduce that $\bar{t}=\bar{s}=1$. Thus $\bar{u}=u$ and $J(u)=m$.

\section{Existence of sign-changing solution}

In this section, we mainly prove that the minimizer $u$ obtained in Lemma 2.5 is indeed a sign-changing solution of (1.1), that is, $J^{\prime}(u)=0$. 
Proof of Theorem 1.4 Using the quantitative deformation lemma [30], Lemma 2.3, we prove that $J^{\prime}(u)=0$. It is clear that $\left\langle J^{\prime}(u), u^{+}\right\rangle=\left\langle J^{\prime}(u), u^{-}\right\rangle=0$. It follows from Lemma 2.3 that, for $(t, s) \in \mathbb{R}_{+} \times \mathbb{R}_{+}$and $(t, s) \neq(1,1)$,

$$
J\left(t u^{+}+s u^{-}\right)<J\left(u^{+}+u^{-}\right)=m .
$$

If $J^{\prime}(u) \neq 0$, then there exist $\delta>0$ small and $\varepsilon_{0}>0$ such that, for all $\|v-u\| \leq 3 \delta$,

$$
\left\|J^{\prime}(v)\right\| \geq \varepsilon_{0}
$$

Let $D:=(1-\delta /\|u\|, 1+\delta /\|u\|) \times(1-\delta /\|u\|, 1+\delta /\|u\|)$ and $h(t, s)=t u^{+}+s u^{-}$. It follows from Lemma 2.3 again that

$$
\bar{m}:=\max _{\partial D} J \circ h<m .
$$

By [30], Lemma 2.3, for $\varepsilon:=\min \left\{(m-\bar{m}) / 2, \varepsilon_{0} \delta / 8\right\}$ and $S:=B(u, \delta)$, there is a deformation $\eta$ such that

(a) $\eta(1, u)=u$ if $u \notin J^{-1}([m-2 \varepsilon, m+2 \varepsilon]) \cap S_{2 \delta}$;

(b) $\eta\left(1, J^{m+\varepsilon} \cap S\right) \subset J^{m-\varepsilon}$;

(c) $J(\eta(1, u)) \leq J(u)$ for all $u \in E$.

For $(t, s) \in \bar{D},\|h(t, s)-u\|^{2}=(t-1)^{2}\left\|u^{+}\right\|^{2}+(s-1)^{2}\left\|u^{-}\right\|^{2} \leq \delta^{2}$, then $h(t, s) \in S$ for all $(t, s) \in \bar{D}$. On the other hand, $\max _{\bar{D}} J(h(t, s))=m$, therefore, from (b) we have

$$
\max _{(t, s) \in \bar{D}} J(\eta(1, h(t, s)))<m
$$

We prove that $\eta(1, h(D)) \cap M \neq \emptyset$, contradicting the definition of $m$. Let us define $\gamma(t, s):=$ $\eta(1, h(t, s))$ and

$$
\begin{aligned}
& \Psi_{0}(t, s):=\left(J^{\prime}(h(t, s)) u^{+}, J^{\prime}(h(t, s)) u^{-}\right)=\left(J^{\prime}\left(t u^{+}+s u^{-}\right) u^{+}, J^{\prime}\left(t u^{+}+s u^{-}\right) u^{-}\right), \\
& \Psi_{1}(t, s):=\left(\frac{1}{t} J^{\prime}(\gamma(t, s)) \gamma^{+}(t, s), \frac{1}{s} J^{\prime}(\gamma(t, s)) \gamma^{-}(t, s)\right) .
\end{aligned}
$$

Since $f: \mathbb{R} \rightarrow \mathbb{R}$ is continuous, $\Psi_{0}: \bar{D} \rightarrow \mathbb{R}^{2}$ is continuous. Then we cannot directly compute the topology degree $\operatorname{deg}\left(\Psi_{0}, D, 0\right)$. Now, we define the operator as follows: $\Phi(t, s):=$ $\left(\phi_{1}(t, s), \phi_{2}(t, s)\right)$ where

$$
\left\{\begin{array}{l}
\phi_{1}(t, s)=-a_{1} t^{2}\left(t^{2}-1\right), \\
\phi_{2}(t, s)=-b_{1} s^{2}\left(s^{2}-1\right) .
\end{array}\right.
$$

Then $\Phi$ has unique zero $(t, s)=(1,1)$. Let us take a homotopy operator

$$
H(\tau,(t, s))=\tau \Psi_{0}+(1-\tau) \Phi
$$

It is easy to see that $H(\tau,(1,1))=0$. Then $H(1,(t, s))=\Psi_{0}(t, s)$ and $H(0,(t, s))=\Phi(t, s)$. Moreover, $H:[0,1] \times \bar{D}$ is continuous. We will show that $H(\tau,(t, s)) \neq 0$ on $[0,1] \times \partial D$. In fact, if $\tau=1$, according to Lemma 2.2 we see that $(1,1)$ is the unique zero point of 
$H(0,(t, s))$. If $\tau=0$, from the definition of $\Phi$, the conclusion is obvious. In the following, we may assume that $\tau \in(0,1)$. It is easy to see that for any $\tau \in(0,1),(1,1)$ is a zero point of $H(\tau,(t, s))$. Therefore, we need only to prove that the zero point of $H(\tau,(t, s))$ is unique in $\bar{D}$. If $\left(t_{0}, s_{0}\right) \in \bar{D}$ is a zero point of $H(\tau,(t, s))$, that is,

$$
\begin{aligned}
& a_{1} \tau t_{0}^{2}+a_{1}(1-\tau) t_{0}^{2}\left(1-t_{0}^{2}\right)+\tau a_{2} t_{0}^{4}+\tau A t_{0}^{2} s_{0}^{2} \\
& -\tau \int_{\mathbb{R}^{N}}\left[K f\left(t_{0} u^{+}\right)\left(t_{0} u^{+}\right)-\theta V(x)\left|t_{0} u^{+}\right|^{2}\right]=0
\end{aligned}
$$

and

$$
\begin{aligned}
& b_{1} \tau s_{0}^{2}+b_{1}(1-\tau) s_{0}^{2}\left(1-s_{0}^{2}\right)+b_{2} \tau s_{0}^{4}+A \tau t_{0}^{2} s_{0}^{2} \\
& \quad-\tau \int_{\mathbb{R}^{N}}\left[K f\left(s_{0} u^{-}\right)\left(s_{0} u^{-}\right)-\theta V(x)\left|s_{0} u^{-}\right|^{2}\right]=0 .
\end{aligned}
$$

We may assume that $t_{0} \leq s_{0}$. Then from (3.5), we have

$$
b_{1}\left[\frac{\tau}{s_{0}^{2}}-\frac{(1-\tau)\left(s_{0}^{2}-1\right)}{s_{0}^{2}}\right]+b_{2} \tau+A \tau \geq \tau \int_{\mathbb{R}^{N}} \frac{K f\left(s_{0} u^{-}\right)-\theta s_{0} V(x)\left(u^{-}\right)^{2}}{s_{0}^{3}\left(u^{-}\right)^{3}}\left(u^{-}\right)^{4} .
$$

On the other hand, it follows from $u \in M$ that

$$
b_{1}+b_{2}+A=\int_{\mathbb{R}^{3}}\left[K f\left(u^{-}\right) u^{-}-\theta V(x)\left(u^{-}\right)^{2}\right] .
$$

Therefore,

$$
\begin{aligned}
b_{1}\left[\frac{1}{s_{0}^{2}}-1\right] & =b_{1}\left[\frac{\tau}{s_{0}^{2}}-\frac{(1-\tau)\left(s_{0}^{2}-1\right)}{s_{0}^{2}}-\tau\right] \\
& \geq \tau \int_{\mathbb{R}^{N}}\left\{\frac{K f\left(s_{0} u^{-}\right)-\theta s_{0} V(x)\left(u^{-}\right)^{2}}{s_{0}^{3}\left(u^{-}\right)^{3}}\left(u^{-}\right)^{4}-\left[K f\left(u^{-}\right) u^{-}-\theta V(x)\left(u^{-}\right)^{2}\right]\right\} .
\end{aligned}
$$

Hence, we have from the condition $\left(\mathrm{f}_{3}\right)$ that $s_{0} \leq 1$. In the following, we prove that $t_{0} \geq 1$. In fact from (3.4) and $0<t_{0} \leq s_{0}$, we have

$$
a_{1}\left[\frac{\tau}{t_{0}^{2}}-\frac{(1-\tau)\left(t_{0}^{2}-1\right)}{t_{0}^{2}}\right]+a_{2} \tau+A \tau \leq \tau \int_{\mathbb{R}^{N}} \frac{K f\left(t_{0} u^{+}\right)-\theta V(x) t_{0} u^{+}}{t_{0}^{3}\left(u^{+}\right)^{3}}\left(u^{+}\right)^{4} .
$$

Since $u \in M$, we have from (3.6)

$$
\begin{aligned}
a_{1}\left[\frac{1}{t_{0}^{2}}-1\right] & =a_{1}\left[\frac{\tau}{t_{0}^{2}}-\frac{(1-\tau)\left(t_{0}^{2}-1\right)}{t_{0}^{2}}-\tau\right] \\
& \leq \tau \int_{\mathbb{R}^{N}} \frac{K f\left(t_{0} u^{+}\right)-\theta V(x) t_{0} u^{+}}{t_{0}^{3}\left(u^{+}\right)^{3}}\left(u^{+}\right)^{4}-\left[K f\left(u^{+}\right) u^{+}-\theta V(x)\left(u^{+}\right)^{2}\right] .
\end{aligned}
$$

Therefore, condition $\left(\mathrm{f}_{3}\right)$ implies that $t_{0} \geq 1$ and then $s_{0}=t_{0}=1$. Hence for $(s, t) \in \partial D$, we obtain $H(\tau,(t, s)) \neq 0$ for all $\tau \in[0,1]$. Therefore, the homotopy invariance property of Brouwer's degree implies that $\operatorname{deg}(H(\tau,(\cdot, \cdot)), D, 0)=$ constant for $\tau \in[0,1]$ and then

$$
\operatorname{deg}\left(\Psi_{0}, D, 0\right)=\operatorname{deg}(H(1,(\cdot, \cdot)), D, 0)=\operatorname{deg}(H(0,(\cdot, \cdot)), D, 0)=\operatorname{deg}(\Phi, D, 0) .
$$


Hence, we need only to compute $\operatorname{deg}(\Phi, D, 0)$. Note that $\Phi \in C^{1}$, and $\Phi$ has an isolated zero point $(1,1)$ in $D$. Since

$$
\begin{aligned}
& \frac{\partial \phi_{1}}{\partial t}(1,1)=-2 a_{1}, \quad \frac{\partial \phi_{1}}{\partial s}(1,1)=0, \\
& \frac{\partial \phi_{2}}{\partial t}(1,1)=0, \quad \frac{\partial \phi_{2}}{\partial s}(1,1)=-2 b_{1},
\end{aligned}
$$

we have

$$
\left|\begin{array}{cc}
-2 a_{1} & 0 \\
0 & -2 b_{1}
\end{array}\right|=4 a_{1} b_{1}>0 .
$$

Then according to the definition of the topological degree, we have

$$
\operatorname{deg}(\Phi, D, 0)=1
$$

On the other hand, it follows from (3.2) that $h=\gamma$ on $\partial D$. Consequently, we obtain $\operatorname{deg}\left(\Psi_{1}, D, 0\right)=\operatorname{deg}\left(\Psi_{0}, D, 0\right)=1$ from the property of the topological degree. Therefore, $\Psi_{1}\left(t_{0}, s_{0}\right)=0$ for some $\left(t_{0}, s_{0}\right) \in D$, that is, $\eta\left(1, h\left(t_{0}, s_{0}\right)\right)=\gamma\left(t_{0}, s_{0}\right) \in M$, which is a contradiction with $\eta(1, h(D)) \cap M \neq \emptyset$. From this, $u$ is a critical point of $J$, and then it is a sign-changing solution for problem (1.1).

\section{Competing interests}

The authors declare that they have no competing interests.

\section{Authors' contributions}

All authors typed, read, and approved the final manuscript.

\section{Acknowledgements}

The authors thank the anonymous referees for their valuable suggestions and comments, by which the paper was revised. This work was partially supported by National Natural Science Foundation of China (Grant Nos. 11301313, 11101250), Science Council of Shanxi Province (2013021001-4).

Received: 7 November 2016 Accepted: 1 December 2016 Published online: 09 December 2016

\section{References}

1. Benci, V, Fortunato, D: An eigenvalue problem for the Schrödinger-Maxwell equations. Topol. Methods Nonlinear Anal. 11(2), 283-293 (1998)

2. Kirchhoff, GR: Mechanik. Teubner, Leipzig (1883)

3. Barrios, B, Peral, I, Vita, S: Some remarks about the summability of nonlocal nonlinear problems. Adv. Nonlinear Anal. 4(2), 91-107 (2015)

4. Bisci, GM, Rădulescu, VD: Ground state solutions of scalar field fractional Schrödinger equations. Calc. Var. Partial Differ. Equ. 54(3), 2985-3008 (2015)

5. Dávila, J, del Pino, M, Dipierro, S, Valdinoci, E: Concentration phenomena for the nonlocal Schrödinger equation with Dirichlet datum. Anal. PDE 8(5), 1165-1235 (2015)

6. Bisci, GM, Radulescu, VD, Servadei, R: Variational Methods for Nonlocal Fractional Problems. Encyclopedia of Mathematics and Its Applications, vol. 162. Cambridge University Press, Cambridge (2016). With a foreword by Jean Mawhin

7. Bisci, GM, Repovš, D: Existence and localization of solutions for nonlocal fractional equations. Asymptot. Anal. 90(3-4), 367-378 (2014)

8. Pucci, $P$, Xiang, $M, Z$ Zhang, B: Existence and multiplicity of entire solutions for fractional $p$-Kirchhoff equations. Adv. Nonlinear Anal. 5(1), 27-55 (2016)

9. Zhang, X, Zhang, B, Repovš, D: Existence and symmetry of solutions for critical fractional Schrödinger equations with bounded potentials. Nonlinear Anal. 142, 48-68 (2016)

10. Azzollini, A, Pomponio, A: On a 'zero mass' nonlinear Schrödinger equation. Adv. Nonlinear Stud. 7(4), 599-627 (2007)

11. Li, F, Li, Y, Shi, J: Existence of positive solutions to Schrödinger-Poisson type systems with critical exponent. Commun. Contemp. Math. 16(6), 1450036 (2014)

12. Li, G, Peng, S, Yan, S: Infinitely many positive solutions for the nonlinear Schrödinger-Poisson system. Commun. Contemp. Math. 12(6), 1069-1092 (2010) 
13. Wang, Z, Zhou, H-S: Positive solution for a nonlinear stationary Schrödinger-Poisson system in $\mathbb{R}^{3}$. Discrete Contin. Dyn. Syst. 18(4), 809-816 (2007)

14. Zhao, L, Liu, H, Zhao, F: Existence and concentration of solutions for the Schrödinger-Poisson equations with steep well potential. J. Differ. Equ. 255(1), 1-23 (2013)

15. Duan, Y, Tang, C-L: Multiple positive solutions for superlinear Kirchhoff type problems on $\mathbb{R}^{N}$. Electron. J. Differ. Equ. 2015, 316 (2015)

16. Fiscella, A, Valdinoci, E: A critical Kirchhoff type problem involving a nonlocal operator. Nonlinear Anal. 94, 156-170 (2014)

17. Li, Y, Li, F, Shi, J: Existence of a positive solution to Kirchhoff type problems without compactness conditions. J. Differ. Equ. 253(7), 2285-2294 (2012)

18. Li, Y, Li, F, Shi, J: Existence of positive solutions to Kirchhoff type problems with zero mass. J. Math. Anal. Appl. 410(1), $361-374(2014)$

19. Liu, J, Liao, J-F, Tang, C-L: The existence of a ground-state solution for a class of Kirchhoff-type equations in $\mathbb{R}^{N}$. Proc. R. Soc. Edinb., Sect. A 146(2), 371-391 (2016)

20. Liu, J, Liao, J-F, Tang, C-L: A positive ground state solution for a class of asymptotically periodic Schrödinger equations. Comput. Math. Appl. 71(4), 965-976 (2016)

21. Zhao, G, Zhu, X, Li, Y: Existence of infinitely many solutions to a class of Kirchhoff-Schrödinger-Poisson system. Appl. Math. Comput. 256, 572-581 (2015)

22. Liang, Z, Xu, J, Zhu, X: Revisit to sign-changing solutions for the nonlinear Schrödinger-Poisson system in $\mathbb{R}^{3}$. J. Math. Anal. Appl. 435(1), 783-799 (2016)

23. Shuai, W, Wang, Q: Existence and asymptotic behavior of sign-changing solutions for the nonlinear Schrödinger-Poisson system in $\mathbb{R}^{3}$. Z. Angew. Math. Phys. 66(6), 3267-3282 (2015)

24. Wang, Z, Zhou, H-S: Sign-changing solutions for the nonlinear Schrödinger-Poisson system in $\mathbb{R}^{3}$. Calc. Var. Partial Differ. Equ. 52(3-4), 927-943 (2015)

25. Shuai, W: Sign-changing solutions for a class of Kirchhoff-type problem in bounded domains. J. Differ. Equ. 259(4) 1256-1274 (2015)

26. Qin, D, Tang, X: Time-harmonic Maxwell equations with asymptotically linear polarization. Z. Angew. Math. Phys. 67(3), Article 39 (2016)

27. Tang, XH, Cheng, B: Ground state sign-changing solutions for Kirchhoff type problems in bounded domains. J. Differ. Equ. 261(4), 2384-2402 (2016)

28. Ambrosetti, A, Felli, V, Malchiodi, A: Ground states of nonlinear Schrödinger equations with potentials vanishing at infinity. Atti Accad. Naz. Lincei Cl. Sci. Fis. Mat. Natur. Rend. Lincei (9) Mat. Appl. 15(2), 81-86 (2004)

29. Miranda, C: Un'osservazione su un teorema di Brouwer. Boll. Unione Mat. Ital. (2) 3, 5-7 (1940)

30. Willem, M: Minimax Theorems. Progress in Nonlinear Differential Equations and Their Applications, vol. 24. Birkhäuser Boston, Boston (1996)

\section{Submit your manuscript to a SpringerOpen ${ }^{\circ}$ journal and benefit from:}

- Convenient online submission

- Rigorous peer review

- Immediate publication on acceptance

- Open access: articles freely available online

- High visibility within the field

- Retaining the copyright to your article 